\title{
All Harmonic Numbers Less than $10^{14}$
}

\author{
Takeshi Goto* and Katsuyuki OKeyA ${ }^{\dagger}$ \\ * Department of Mathematics, Faculty of Science and Technology \\ Tokyo University of Science, Noda, Chiba, 278-8510, Japan \\ E-mail: goto_takeshi@ma.noda.tus.ac.jp \\ †Hitachi, Ltd., Systems Development Laboratory \\ 1099, Ohzenji, Asao-ku, Kawasaki, 215-0013, Japan \\ E-mail: katsuyuki.okeya.ue@hitachi.com
}

Received October 31, 2006

Revised March 22, 2007

\begin{abstract}
A positive integer $n$ is said to be harmonic if the harmonic mean $H(n)$ of its positive divisors is an integer. Ore proved that every perfect number is harmonic and conjectured that there exist no odd harmonic numbers greater than 1 . In this article, we give the list of all harmonic numbers up to $10^{14}$. In particular, we find that there exist no nontrivial odd harmonic numbers less than $10^{14}$.
\end{abstract}

Key words: harmonic number, perfect number, Ore's conjecture

\section{Introduction}

A positive integer $n$ is said to be perfect if $\sigma(n)=2 n$, where $\sigma(n)$ denotes the sum of the positive divisors of $n$. It is an open problem whether or not an odd perfect number exists. In 1948, Ore [7] introduced the concept of harmonic numbers. In general, the harmonic mean of positive numbers $a_{1}, \ldots, a_{k}$ is defined by

$$
\left(\sum_{i=1}^{k} \frac{1}{a_{i}}\right)^{-1}
$$

A positive integer $n$ is said to be harmonic if the harmonic mean of its positive divisors

$$
H(n)=\frac{n \tau(n)}{\sigma(n)}
$$

is an integer, where $\tau(n)$ denotes the number of the positive divisors of $n$. We call 1 the trivial harmonic number. Ore proved that every perfect number is harmonic. Many harmonic numbers are known; however, no nontrivial odd harmonic numbers have been discovered. Ore conjectured that every nontrivial harmonic number is even. If Ore's conjecture is true, then it follows that no odd perfect numbers exist.

Ore listed all harmonic numbers up to $10^{4}$ and this list was extended by Garcia [4] to $10^{7}$, by Cohen [2] to $2 \cdot 10^{9}$, and by Sorli [8] to $10^{12}$. They used the algorithm described in [4]. In this article, we give the list of all harmonic numbers up to $10^{14}$ using an improved algorithm.

TheOREM 1.1. Let $n$ be harmonic numbers less than $10^{14}$. Then $n$ is one of the 937 numbers in $\S 5$. 
In particular, we find that there exist no nontrivial odd harmonic numbers less than $10^{14}$. Note that Sorli [8] showed that there exist no nontrivial odd harmonic numbers less than $10^{15}$ by another method. Cohen and Sorli [3] introduced the concept of harmonic seeds.

Definition. Let $d$ be a divisor of an integer $n$. We call $d$ a unitary divisor of $n$ and $n$ a unitary multiple of $d$ if $(d, n / d)=1$. A harmonic number is called harmonic seed if it does not have a smaller proper unitary divisor which is harmonic (we call a unitary divisor $d$ proper if $d>1$ ).

Cohen and Sorli [3] gave the list of all harmonic seeds up to $10^{12}$, and this list was extended by Sorli [8] to $10^{15}$. Note that our list is consistent with these lists. Sorli's list contains no odd harmonic seeds greater than 1. Since every harmonic number is a unitary multiple of a seed, it follows that there exist no nontrivial odd harmonic numbers less than $10^{15}$.

Theorem 1.1 is a consequence of the following two lemmas. The lists mentioned in the statements are available on the webpage http://www.ma.noda.tus.ac.jp/ $\mathrm{u} / \mathrm{tg} / \mathrm{html} / \mathrm{harmonic}-\mathrm{e} . \mathrm{html}$.

LEMMA 1.2. Let $n$ be harmonic and $H(n)^{4.55}>n$. Then $n$ is one of the 1643 numbers in the list which is available on the webpage.

LEMMA 1.3. Let $n$ be harmonic and $H(n) \leq 1200$. Then $n$ is one of the 1376 numbers in the list which is available on the webpage.

Proof of Theorem 1.1. Let

$$
\begin{aligned}
& \mathcal{H}=\{n \in \mathbb{N} \mid H(n) \in \mathbb{N}\}, \\
& \mathcal{H}_{1}=\left\{n \in \mathcal{H} \mid n<10^{14}\right\}, \\
& \mathcal{H}_{2}=\left\{n \in \mathcal{H} \mid H(n)^{4.55}>n\right\}, \\
& \mathcal{H}_{3}=\{n \in \mathcal{H} \mid H(n) \leq 1200\} .
\end{aligned}
$$

We can easily see that $\mathcal{H}_{1} \subset \mathcal{H}_{2} \cap \mathcal{H}_{3}$. Indeed, suppose that $n \in \mathcal{H}_{1}$. If $n \notin \mathcal{H}_{3}$, then $H(n)^{4.55}>1200^{4.55}>10^{14}>n$, and hence $n \in \mathcal{H}_{2}$. By Lemmas 1.2 and 1.3, the finite sets $\mathcal{H}_{2}$ and $\mathcal{H}_{3}$ are known. Therefore we can give the set $\mathcal{H}_{1}$.

Note that Lemma 1.2 is an example of the following theorem.

THEOREM 1.4. For any real number $\alpha$, there exist only finitely many positive integers $n$ satisfying $H(n)^{\alpha}>n$.

In $\S 2$, we give a proof of Theorem 1.4. The proof describes the algorithm to show Lemma 1.2.

REMARK. It is well known that a harmonic mean is equal to or less than a geometric mean. Since the geometric mean of positive divisors of $n$ is $\sqrt{n}$, there exist no harmonic numbers $n$ satisfying $H(n)^{2}>n$. 
Shibata and the first author [5] gave the list of all harmonic numbers $n$ with $H(n) \leq 300$. Lemma 1.3 is the extended result. In $\S 3$, we discuss the algorithm to show it.

\section{Proof of Theorem 1.4 and Lemma 1.2}

Proof of Theorem 1.4. From the remark after Theorem 1.4, we may assume that $\alpha>2$. Now, let us fix a real number $\alpha$ and define $f(\alpha, n)=H(n)^{\alpha} / n$. Then $H(n)^{\alpha}>n$ if and only if $f(\alpha, n)>1$. Note that $f$ is multiplicative in the second variable, that is, $f(\alpha, n m)=f(\alpha, n) f(\alpha, m)$ when $(n, m)=1$. For a prime $p$ and a positive integer $e$,

$$
f\left(\alpha, p^{e}\right)=\frac{p^{(\alpha-1) e}(e+1)^{\alpha}}{\left(p^{e}+p^{e-1}+\cdots+1\right)^{\alpha}} .
$$

Since $\alpha>2$, it is clear that $f\left(\alpha, p^{e}\right)$ is monotone decreasing as a function of $p$ and of $e$ for sufficiently large $p$ and $e$. Furthermore, we have $\lim _{p \rightarrow \infty} f\left(\alpha, p^{e}\right)=0$ and $\lim _{e \rightarrow \infty} f\left(\alpha, p^{e}\right)=0$. Hence there are only finitely many prime powers $p^{e}$ satisfying $f\left(\alpha, p^{e}\right)>1$. Let $\mathscr{L}$ be the set of integers whose all prime components satisfy this condition. Since $\mathscr{L}$ is finite, there exists the maximum value $\max _{n \in \mathscr{L}} f(\alpha, n)$. Let $A$ be this maximum value. There are also only finitely many prime powers $q^{f}$ satisfying $f\left(\alpha, q^{f}\right)>1 / A$. Let $\mathscr{L}^{\prime}$ be the set of integers whose all prime components satisfy this condition, and $\mathscr{M}$ be the set of integers $n$ satisfying the required condition $f(\alpha, n)>1$. It is easy to show that $\mathscr{M} \subset \mathscr{L}^{\prime}$. Since $\mathscr{L}^{\prime}$ is finite, $\mathscr{M}$ is also finite, and hence the proof is complete.

In the rest of this section, we demonstrate this procedure for the case of $\alpha=$ 4.55. We first give some useful lemmas.

LeMma 2.1. If $p>((e+2) /(e+1))^{\alpha}$, then $f\left(\alpha, p^{e}\right)>f\left(\alpha, p^{e+1}\right)$. In particular, if $p>(3 / 2)^{\alpha}$, then $f\left(\alpha, p^{e}\right)>f\left(\alpha, p^{e+1}\right)$ for any positive integer $e$.

Proof. Suppose that $p>((e+2) /(e+1))^{\alpha}$. Then we have

$$
\begin{aligned}
\frac{f\left(\alpha, p^{e}\right)}{f\left(\alpha, p^{e+1}\right)} & =\frac{p^{(\alpha-1) e}(p-1)^{\alpha}(e+1)^{\alpha}}{\left(p^{e+1}-1\right)^{\alpha}} \cdot \frac{\left(p^{e+2}-1\right)^{\alpha}}{p^{(\alpha-1)(e+1)}(p-1)^{\alpha}(e+2)^{\alpha}} \\
& =p\left(\frac{p^{e+2}-1}{p^{e+2}-p}\right)^{\alpha}\left(\frac{e+1}{e+2}\right)^{\alpha}>p\left(\frac{e+1}{e+2}\right)^{\alpha}>1,
\end{aligned}
$$

and hence $f\left(\alpha, p^{e}\right)>f\left(\alpha, p^{e+1}\right)$.

Lemma 2.2. Let $p, q$ be primes and $\alpha-1 \leq p<q$. Then $f(\alpha, p)>f(\alpha, q)$.

Proof. By a direct calculation, we have

$$
\frac{\partial f(\alpha, p)}{\partial p}=\frac{2^{\alpha} p^{\alpha-2}}{(p+1)^{\alpha+1}}(\alpha-p-1) .
$$

Hence the lemma holds. 
Let $f(n)=f(4.55, n)=H(n)^{4.55} / n$. By a direct calculation, we have the following table.

Table 1. Values of $f\left(p^{e}\right)$

\begin{tabular}{|c|c||c|c||c|c||c|c|}
\hline$p^{e}$ & $f\left(p^{e}\right)$ & $p^{e}$ & $f\left(p^{e}\right)$ & $p^{e}$ & $f\left(p^{e}\right)$ & $p^{e}$ & $f\left(p^{e}\right)$ \\
\hline 2 & 1.85114 & $2^{9}$ & 2.97148 & $3^{4}$ & 3.01135 & 7 & 1.82274 \\
$2^{2}$ & 2.90414 & $2^{10}$ & 2.28725 & $3^{5}$ & 2.27237 & $7^{2}$ & 1.52008 \\
$2^{3}$ & 3.92759 & $2^{11}$ & 1.69723 & $3^{6}$ & 1.52109 & $7^{3}$ & 0.79486 \\
$2^{4}$ & 4.66921 & $2^{12}$ & 1.22077 & $3^{7}$ & 0.92960 & 11 & 1.43336 \\
$2^{5}$ & 4.97589 & $2^{13}$ & 0.85492 & 5 & 2.04381 & $11^{2}$ & 0.79664 \\
$2^{6}$ & 4.83980 & 3 & 2.10908 & $5^{2}$ & 2.22788 & 13 & 1.28618 \\
$2^{7}$ & 4.36412 & $3^{2}$ & 3.09044 & $5^{3}$ & 1.60208 & 17 & 1.06240 \\
$2^{8}$ & 3.69607 & $3^{3}$ & 3.39892 & $5^{4}$ & 0.87927 & 19 & 0.97628 \\
\hline
\end{tabular}

By Lemma 2.2 and Table $1, f(p)$ is monotone decreasing for $p \geq 3$. By Lemma 2.1, we have

- $f\left(2^{5}\right) \geq f\left(2^{e}\right)$ for $e \geq 5$,

- $f\left(3^{3}\right) \geq f\left(3^{e}\right)$ for $e \geq 3$,

- $f\left(5^{2}\right) \geq f\left(5^{e}\right)$ for $e \geq 2$,

- $f(p) \geq f\left(p^{e}\right)$ for $e \geq 1, p \geq 7$.

Hence it follows that

$$
\mathscr{L}=\left\{\begin{array}{l|l}
2^{\varepsilon_{1}} 3^{\varepsilon_{2}} 5^{\varepsilon_{3}} 7^{\varepsilon_{4}} 11^{\varepsilon_{5}} 13^{\varepsilon_{6}} 17^{\varepsilon_{7}} \mid \begin{array}{l}
0 \leq \varepsilon_{1} \leq 12,0 \leq \varepsilon_{2} \leq 6,0 \leq \varepsilon_{3} \leq 3, \\
0 \leq \varepsilon_{4} \leq 2,0 \leq \varepsilon_{5}, \varepsilon_{6}, \varepsilon_{7} \leq 1
\end{array}
\end{array}\right\},
$$

and $A:=\max _{n \in \mathscr{L}} f(n)=f\left(2^{5} \cdot 3^{3} \cdot 5^{2} \cdot 7 \cdot 11 \cdot 13 \cdot 17\right)<4.9759 \cdot 3.399 \cdot 2.2279$. $1.8228 \cdot 1.4334 \cdot 1.2862 \cdot 1.0625<134.55$. Therefore, if $f(n)>1$, then every prime component $p^{e}$ of $n$ satisfies $f\left(p^{e}\right)>1 / A>1 / 134.55>0.007432$. A computer search showed that $\mathscr{L}^{\prime}$ is the set of integers whose all prime components are in the set

$$
\left\{\begin{array}{l}
2^{e}(1 \leq e \leq 23), 3^{e}(1 \leq e \leq 13), 5^{e}(1 \leq e \leq 8) \\
7^{e}(1 \leq e \leq 7), 11^{e}(1 \leq e \leq 5), 13^{e}, 17^{e}, 19^{e}(1 \leq e \leq 4) \\
23^{e}, 29^{e}, 31^{e}, 37^{e}(1 \leq e \leq 3), p^{2}(41 \leq p \leq 137), p(41 \leq p \leq 3137)
\end{array}\right\}
$$

We have to select harmonic numbers from $\mathscr{L}^{\prime}$. Using the method of Garcia [4], we can do it and show Lemma 1.2. Using Mathematicaß and a machine with a processor Pentium M $1.2 \mathrm{GHz}$, the authors needed about 3 hours to do this computation. In the case of $\alpha=4$, we needed only ten seconds. These data show that the amount of computation increases very rapidly with $\alpha$. 
Table 2. More values of $f\left(p^{e}\right)$

\begin{tabular}{|c|c||c|c||c|c||c|c|}
\hline$p^{e}$ & $f\left(p^{e}\right)$ & $p^{e}$ & $f\left(p^{e}\right)$ & $p^{e}$ & $f\left(p^{e}\right)$ & $p^{e}$ & $f\left(p^{e}\right)$ \\
\hline $2^{23}$ & 0.00969 & $11^{5}$ & 0.01397 & $23^{3}$ & 0.03684 & $41^{2}$ & 0.07880 \\
$2^{24}$ & 0.00583 & $11^{6}$ & 0.00256 & $23^{4}$ & 0.00442 & $41^{3}$ & 0.00711 \\
$3^{13}$ & 0.01625 & $13^{4}$ & 0.03684 & $29^{3}$ & 0.01917 & $43^{2}$ & 0.07202 \\
$3^{14}$ & 0.00741 & $13^{5}$ & 0.00649 & $29^{4}$ & 0.00182 & $137^{2}$ & 0.00763 \\
$5^{8}$ & 0.02037 & $17^{4}$ & 0.01376 & $31^{3}$ & 0.01586 & $139^{2}$ & 0.00742 \\
$5^{9}$ & 0.00658 & $17^{5}$ & 0.00185 & $31^{4}$ & 0.00141 & 3137 & 0.00745 \\
$7^{7}$ & 0.00774 & $19^{4}$ & 0.00908 & $37^{3}$ & 0.00956 & 3163 & 0.00739 \\
$7^{8}$ & 0.00188 & $19^{5}$ & 0.00109 & $37^{4}$ & 0.00071 & & \\
\hline
\end{tabular}

\section{Algorithm to show Lemma 1.3}

We first summarize the algorithm described in [5]. The algorithm is to find all harmonic numbers $n$ satisfying $H(n)=c$ for a given integer $c$. In this section, we say that an integer $n$ has a type of exponents $\left(e_{1}, \ldots, e_{r}\right)$ when the factorization of $n$ is $p_{1}^{e_{1}} \cdots p_{r}^{e_{r}}$ with $e_{1} \geq \cdots \geq e_{r}$. If $n$ has the type of exponents $\left(e_{1}, \ldots, e_{r}\right)$, then

$$
c=H(n) \geq H\left(2^{e_{1}} 3^{e_{2}} \cdots q_{r}^{e_{r}}\right),
$$

where $q_{r}$ is the $r$ th prime (see Lemmas 2.1 and 4.2 in [5]). If $H(n)=c$ and $n$ has the type of exponents $\left(e_{1}, \ldots, e_{r}\right)$, then

$$
S(n):=\frac{\sigma(n)}{n}=\frac{\tau(n)}{H(n)}=\frac{\left(e_{1}+1\right) \cdots\left(e_{r}+1\right)}{c} .
$$

Hence we need to find all integers $n$ which have the type $\left(e_{1}, \ldots, e_{r}\right)$ and satisfy $S(n)=d$ for a given rational number $d$. It is clear that the function $S$ is multiplicative, that is, $S(m n)=S(m) S(n)$ when $(m, n)=1$. Furthermore, if $p, q$ are primes and $p<q$, then

$$
1<\frac{q+1}{q} \leq S\left(q^{e}\right)<S\left(p^{e}\right)<\frac{p}{p-1} .
$$

Hence, if $p$ is a smallest prime dividing $n$, then

$$
d=S(n)<S\left(p^{e_{1}}\right) \cdots S\left(p^{e_{r}}\right)<\left(\frac{p}{p-1}\right)^{r} .
$$

Therefore it is necessary that

$$
p<\frac{\sqrt[r]{d}}{\sqrt[r]{d}-1} .
$$

The basic algorithm to find all integers $n$ which have the type $\left(e_{1}, \ldots, e_{r}\right)$ and satisfy $S(n)=d$, is described as follows. Note that this procedure will end since the number of the second terms decreases for each step of the subroutine. 
Basic algorithm

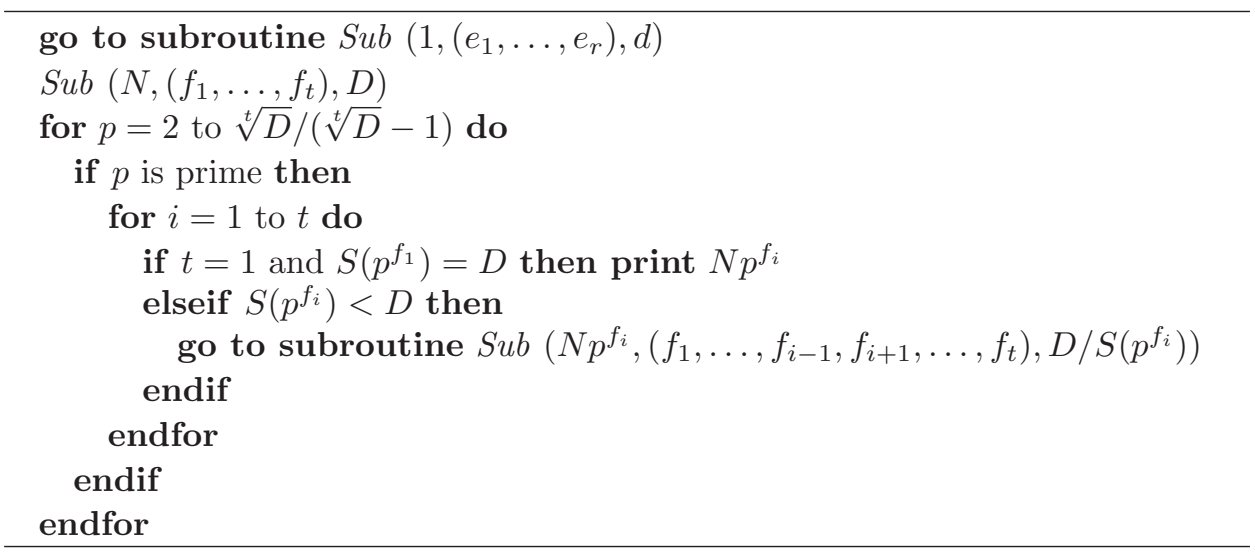

The problem with this algorithm is that the bound $\sqrt[t]{D} /(\sqrt[t]{D}-1)$ is large when $D$ is close to 1 .

Example 1. Let $p_{1}, p_{2}, p_{3}$ be odd primes with $p_{1}<p_{2}<p_{3}$. Consider the equation $H\left(2^{3} p_{1} p_{2} p_{3}\right)=17$. Since $H\left(2^{3}\right)=2^{5} /(3 \cdot 5)$, we have $H\left(p_{1} p_{2} p_{3}\right)=17$. $3 \cdot 5 / 2^{5}$. Therefore $S\left(p_{1} p_{2} p_{3}\right)=2^{8} /(3 \cdot 5 \cdot 17)=1.0039 \cdots$, and the upper bound of $p_{1}$ is a little large. It is about 767 .

Example 2. Let $l, p_{1}, p_{2}$ be odd primes with $p_{1}<p_{2}$. Consider the equation $H\left(2^{l-1} p_{1} p_{2}\right)=2 l$. Since $H\left(2^{l-1}\right)=2^{l-1} l /\left(2^{l}-1\right)$, it follows that $H\left(p_{1} p_{2}\right)=2 l\left(2^{l}-\right.$ $1) /\left(2^{l-1} l\right)=\left(2^{l}-1\right) / 2^{l-2}$. Therefore $S\left(p_{1} p_{2}\right)=2^{l} /\left(2^{l}-1\right)$ and it is very close to 1 , the bound of $p_{1}$ is large. For example, if $l=47$, then the bound is greater than $10^{14}$.

Using the following proposition, we can improve the algorithm.

Proposition 3.1. Suppose that $n$ has a type of exponents $\left(e_{1}, \ldots, e_{r}\right)$ and $H(n)=a / b(a, b \in \mathbb{N},(a, b)=1)$. Let $m$ be the greatest common divisor of $a$ and $\tau(n)\left(=\left(e_{1}+1\right) \cdots\left(e_{r}+1\right)\right)$. Then $a / m$ divides $n$.

Proof. From $H(n)=n \tau(n) / \sigma(n)=a / b$, we have $(a / m) \cdot \sigma(n)=n b \tau(n) / m$. Since $a / m$ is coprime to $b \tau(n) / m$, it follows that $a / m$ divides $n$.

Let us go back to Example 1. From $H\left(2^{3} p_{1} p_{2} p_{3}\right)=17$, we have $H\left(p_{1} p_{2} p_{3}\right)=$ $17 / H\left(2^{3}\right)=(3 \cdot 5 \cdot 17) / 2^{5}$. By Proposition 3.1 , it is necessary that $p_{1} p_{2} p_{3}=3 \cdot 5 \cdot 17$; however, $H\left(2^{3} \cdot 3 \cdot 5 \cdot 17\right) \neq 17$. Hence no solutions exist. This method is often very effective, but sometimes it has the opposite effect.

Example 3. Let $p_{1}, p_{2}$ be odd primes with $p_{1}<p_{2}$. Consider the equation $H\left(5^{100} p_{1}^{2} p_{2}\right)=303$. From the equation, we have $H\left(p_{1}^{2} p_{2}\right)=3 \sigma\left(5^{100}\right) / 5^{100}$. Hence it is necessary that $\sigma\left(5^{100}\right) \mid p_{1}^{2} p_{2}$ by Proposition 3.1. However $\sigma\left(5^{100}\right)$ is a 70 -digit integer, and it is slightly difficult to find a prime factor of the integer*. Hence we

\footnotetext{
*According to the book [1], the factorization of $\sigma\left(5^{100}\right)$ is given by 5937018283241 . $3434487311396589821473854121 \cdot 483593153887747265029536907421$.
} 
should use the upper bound of $p_{1}$. Let $D=S\left(p_{1}^{2} p_{2}\right)$. Then it is necessary that $p_{1}<\sqrt{D} /(\sqrt{D}-1)=4.77 \cdots$, and hence $p_{1}=2$ or 3 . his is contradictory to the condition $\sigma\left(5^{100}\right) \mid p_{1}^{2} p_{2}$ since $\sigma\left(5^{100}\right)$ is not divisible by either 2 or 3 . Therefore no solutions exist.

In this way, we have the following algorithm.

Improved algorithm

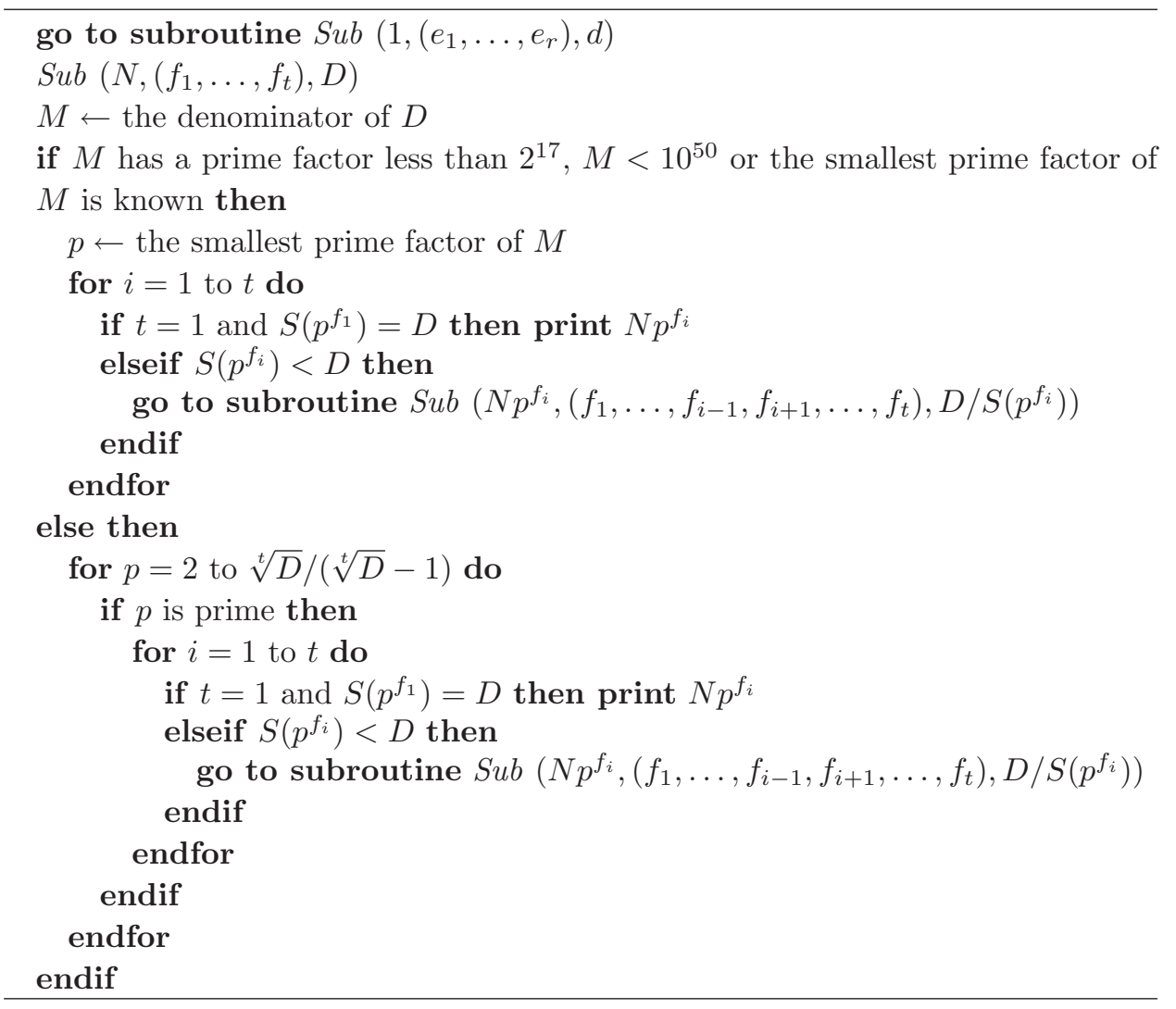

In this algorithm, we factor integers less than $10^{50}$. It is possible to change this value. The authors used UBASIC to factor integers. We can find a prime factor less than $2^{17}$ using the command prmdiv (trial division), and the program MPQX3 (multiple polynomial quadratic sieve, implemented by Y. Kida and A. Yamasaki) enable us to factor 50-digit integers within a few seconds.

Unfortunately, both $a / m$ and $\sqrt[t]{D} /(\sqrt[t]{D}-1)$ are sometimes very large. Consider Example 2 again. From the equation $H\left(2^{l-1} p_{1} p_{2}\right)=2 l$, it follows that $H\left(p_{1} p_{2}\right)=$ $\left(2^{l}-1\right) / 2^{l-2}$. By Proposition 3.1, it is necessary that $2^{l}-1 \mid p_{1} p_{2}$. If $l=47$, then $2^{47}-1 \mid p_{1} p_{2}$ is impossible since $2^{47}-1=2351 \cdot 4513 \cdot 13264529$. When $l$ is large, it is difficult to factor the Mersenne number $2^{l}-1$. Hence the following proposition is useful. 
Proposition $3.2([2],[5])$. Let $p$ be prime. If $H(n)=p, 2 p$ or $3 p$, then $n$ is an even perfect number or $p \mid n$.

From this proposition, it is necessary that $p_{1}=l$ or $p_{2}=l$. Recall that $2^{l}-1 \mid$ $p_{1} p_{2}$. Since $l \nmid 2^{l}-1$ from Fermat's Little Theorem, it is necessary that $2^{l}-1$ is prime and $n=2^{l-1} l\left(2^{l}-1\right)$. However $H(n)$ is not integral for this $n$, and hence no solutions exist.

The following example is one of the most unfortunate cases.

Example 4. Let $p_{1}, p_{2}$ be odd primes with $p_{1}<p_{2}$. Consider the equation $H\left(2^{l-1} p_{1} p_{2} p_{3}\right)=4 l$. From this equation, we have $H\left(p_{1} p_{2} p_{3}\right)=\left(2^{l}-1\right) / 2^{l-3}$ and $S\left(p_{1} p_{2} p_{3}\right)=2^{l} /\left(2^{l}-1\right)$. Both the number $2^{l}-1$ and the upper bound of $p_{1}$ are large. Furthermore we cannot apply Proposition 3.2 in this case. In such a case, we can refer to known prime factors of Mersenne numbers $2^{l}-1$. As of March 2007, at least one prime factor of $2^{l}-1$ for $l<1061$ is known (cf. [1] or recent webpages $^{\dagger}$ ). No factors of $2^{1061}-1$ are known, and hence it is unknown whether or not $H\left(2^{1060} p_{1} p_{2} p_{3}\right)=4 \cdot 1060$ has a solution.

The authors used UBASIC and it took about 10 hours to show Lemma 1.3.

\section{Numerical data}

In this section, we give some interesting examples and numerical data.

Let $x, y$ be harmonic numbers. In this section, we write $x \preceq y$ if $x \neq 1$ and $x$ is a unitary divisor of $y$. If $x \preceq y$ and $x \neq y$, then we write $x \prec y$. Clearly, $x \prec y, y \prec z$ implies $x \prec z$. In other words, the notation $\prec$ is the partial order relationship in the set of harmonic numbers. A harmonic number $n$ is a seed if it is minimal for this order, that is, there exist no harmonic numbers $n^{\prime}$ satisfying $n^{\prime} \prec n$. Every harmonic number is a unitary multiple of a certain harmonic seed. Cohen and Sorli [3] raised following question.

Problem 1. Does every harmonic number have a unique harmonic seed?

From Lemma 1.3, we find that the answer is no. The harmonic number $n_{0}=$ $2^{4} \cdot 3 \cdot 5^{2} \cdot 7^{2} \cdot 19 \cdot 31^{2} \cdot 83 \cdot 331$ with $H\left(n_{0}\right)=525$ has two harmonic seeds $n_{1}=$ $2^{4} \cdot 3 \cdot 7^{2} \cdot 19 \cdot 31^{2} \cdot 83 \cdot 331$ with $H\left(n_{1}\right)=217$, and $n_{2}=2^{4} \cdot 5^{2} \cdot 7^{2} \cdot 19 \cdot 31^{2} \cdot 83 \cdot 331$ with $H\left(n_{2}\right)=350$. In other words, $n_{1}, n_{2} \prec n_{0}$ and there are no harmonic numbers $n$ satisfying $n \prec n_{1}$ or $n \prec n_{2}$. If $m$ is a harmonic number and $n_{0} \prec m$, then $m$ also has two harmonic seeds $n_{1}$ and $n_{2}$. There are many such harmonic numbers: $13 n_{0}, 29 n_{0}, 41 n_{0}$, and so on.

A positive integer $n$ is said to be arithmetic if the arithmetic mean of its positive divisors, $A(n)=\sigma(n) / \tau(n)$, is an integer. Ore conjectured that all harmonic numbers $n$ are perfect or arithmetic; however, he soon found the counterexample $950976(H(950976)=27, A(950976)=105664 / 3)$. Cohen [2] showed that $n$ is arithmetic if $H(n)$ is a prime and $n$ is not an even perfect number. Shibata and

\footnotetext{
${ }^{\dagger}$ See, for example, P. Leyland's page http://www.leyland.vispa.com/numth/factorization/ cunningham/2-.txt.
} 
the first author [5] showed that $n$ is arithmetic if $H(n)$ is the double of a prime. They raised following question (cf. [6, B2]).

Problem 2. Assume that $H(n)$ is the triple of a prime. Is $n$ arithmetic?

From Lemma 1.3, we find that the answer is also no. The harmonic number $n=2^{8} \cdot 7 \cdot 19^{2} \cdot 37 \cdot 73 \cdot 113 \cdot 127$ with $H(n)=3 \cdot 113$ is not arithmetic $(A(n)=$ $221908282624 / 3)$.

Let $N(x)=\#\{n \in \mathbb{N} \mid H(n) \in \mathbb{N}, H(n) \leq x\}$ and $N^{\prime}(x)=\#\{n \in \mathbb{N} \mid n$ is a seed, $H(n) \leq x\}$ for a real number $x$. From Lemma 1.3, we see that $N(1200)=$ $1376, N^{\prime}(1200)=188$.

Problem 3. How does the number $N(x)$ increase when $x$ increases? What is the order of $N(x)$ as $x \rightarrow \infty$ ? How about $N^{\prime}(x)$ ?

The number $N(x)$ (resp. $\left.N^{\prime}(x)\right)$ seems to be close to $x^{1.015}$ (resp. $\left.x^{0.74}\right)$.

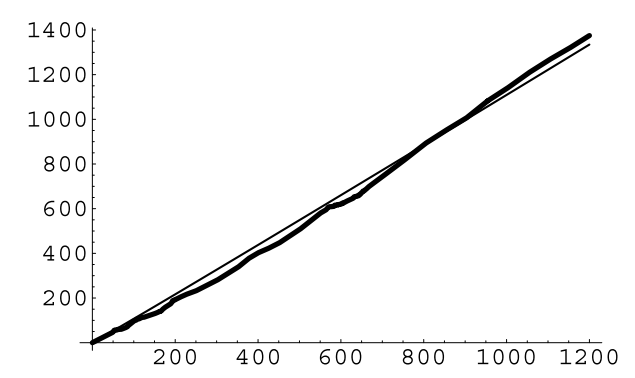

Fig. 1. The graphs of $N(x)$ and $x^{1.015}$

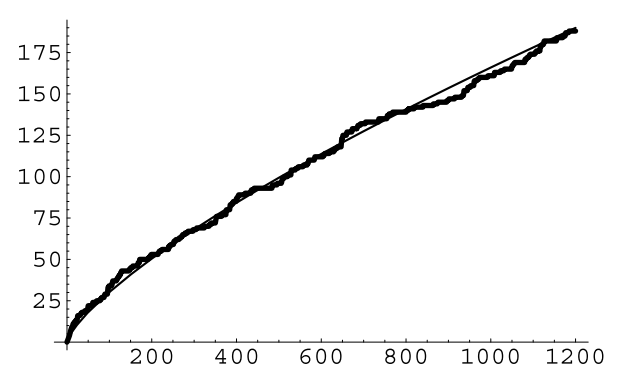

Fig. 2. The graphs of $N^{\prime}(x)$ and $x^{0.74}$

The following problem is also still open.

Problem 4. Are there infinitely many harmonic numbers? How about seeds?

The above graphs are evidence that there seem to exist infinitely many harmonic numbers and seeds. 
Let $M(c)=\#\{n \in \mathbb{N} \mid H(n)=c\}$ and $M^{\prime}(c)=\#\{n \in \mathbb{N} \mid n$ is a seed, $H(n)=c\}$ for an integer $c$. We find that $M(1155)=7$ and $M^{\prime}(648)=5$. In fact,

$$
\begin{aligned}
1155 & =H(30233275380000)=H(30368564724960)=H(32752714995000) \\
& =H(256304066430720)=H(557957132902400)=H(5685642164944896) \\
& =H(16919757239726880), \\
648 & =H(513480135168)=H(1058501001600)=H(1085239701000) \\
& =H(1599300612000)=H(10881843388416) .
\end{aligned}
$$

From Lemma 1.3, if $c \leq 1200$, then $M(c) \leq 7$ and $M^{\prime}(c) \leq 5$.

Problem 5. Can $M(c)$ be arbitrarily large? How about $M^{\prime}(c)$ ?

\section{Table of harmonic numbers}

\begin{tabular}{|c|c|c|c|c|c|c|c|}
\hline$n$ & $H$ & $n$ & $H$ & $n$ & $H$ & $n$ & $H$ \\
\hline 1 & 1 & 753480 & 46 & ${ }^{*} 33550336$ & 13 & 301953024 & 27 \\
\hline${ }^{*} 6$ & 2 & 950976 & 27 & 37035180 & 102 & 318177800 & 73 \\
\hline$* 28$ & 3 & ${ }^{*} 1089270$ & 42 & 44660070 & 82 & 318729600 & 168 \\
\hline 140 & 5 & 1421280 & 47 & *45532800 & 96 & ${ }^{*} 326781000$ & 168 \\
\hline$* 270$ & 6 & 1539720 & 47 & 46683000 & 114 & 400851360 & 184 \\
\hline *496 & 5 & 2178540 & 54 & 50401728 & 53 & 407386980 & 187 \\
\hline${ }^{*} 672$ & 8 & *2229500 & 35 & *52141320 & 108 & 423184320 & 89 \\
\hline${ }^{*} 1638$ & 9 & 2290260 & 41 & 56511000 & 115 & 428972544 & 156 \\
\hline 2970 & 11 & *2457000 & 60 & 69266400 & 105 & 447828480 & 152 \\
\hline$* 6200$ & 10 & 2845800 & 51 & 71253000 & 116 & *459818240 & 96 \\
\hline *8128 & 7 & 4358600 & 37 & 75038600 & 91 & *481572000 & 168 \\
\hline 8190 & 15 & *4713984 & 48 & 80832960 & 85 & 499974930 & 153 \\
\hline 18600 & 15 & 4754880 & 45 & *81695250 & 105 & 500860800 & 176 \\
\hline${ }^{*} 18620$ & 14 & 5772200 & 49 & 90409410 & 83 & 513513000 & 209 \\
\hline 27846 & 17 & ${ }^{*} 6051500$ & 50 & 108421632 & 92 & 526480500 & 145 \\
\hline *30240 & 24 & *8506400 & 49 & 110583200 & 91 & 540277920 & 186 \\
\hline *32760 & 24 & 8872200 & 53 & *115048440 & 78 & 559903400 & 97 \\
\hline 55860 & 21 & 11981970 & 77 & 115462620 & 106 & 623397600 & 189 \\
\hline 105664 & 13 & 14303520 & 86 & 137891520 & 87 & *644271264 & 117 \\
\hline 117800 & 19 & 15495480 & 86 & *142990848 & 120 & 675347400 & 189 \\
\hline 167400 & 27 & 16166592 & 51 & 144963000 & 118 & 714954240 & 200 \\
\hline *173600 & 25 & *17428320 & 96 & 163390500 & 135 & 758951424 & 161 \\
\hline 237510 & 29 & 18154500 & 75 & 164989440 & 140 & 766284288 & 132 \\
\hline 242060 & 26 & *23088800 & 70 & 191711520 & 176 & 819131040 & 188 \\
\hline 332640 & 44 & 23569920 & 80 & 221557248 & 94 & 825120800 & 97 \\
\hline 360360 & 44 & 23963940 & 99 & 233103780 & 107 & 886402440 & 204 \\
\hline 539400 & 29 & 27027000 & 110 & *255428096 & 88 & 900463200 & 195 \\
\hline 695520 & 46 & * 29410290 & 81 & 287425800 & 101 & 995248800 & 189 \\
\hline 726180 & 39 & 32997888 & 84 & 300154400 & 130 & 1047254400 & 184 \\
\hline
\end{tabular}

The table in this section is the list of all harmonic numbers up to $10^{14}$. In this table, harmonic seeds are marked with an asterisk. The authors think that the list can be extended by improving the computer programs. For the most recent results, see the webpage http://www.ma.noda.tus.ac.jp/tg/html/harmonic-e.html. 


\begin{tabular}{|c|c|c|c|c|c|c|c|}
\hline$n$ & $H$ & $n$ & $H$ & $n$ & $H$ & $n$ & $H$ \\
\hline 1162161000 & 215 & 8436460032 & 236 & 30063852000 & 460 & 89526646440 & 404 \\
\hline 1199250360 & 207 & *8589869056 & 17 & 30600708096 & 144 & 93419333280 & 377 \\
\hline 1265532840 & 143 & *8628633000 & 195 & 31638321000 & 275 & 95088913920 & 560 \\
\hline *1307124000 & 240 & 8659696500 & 265 & 31727458560 & 276 & 95300150400 & 598 \\
\hline 1352913408 & 164 & 8696764800 & 191 & 31766716800 & 460 & 97941285120 & 284 \\
\hline 1379454720 & 144 & *8698459616 & 121 & 32950224384 & 258 & 100383241728 & 262 \\
\hline *1381161600 & 240 & 9866368512 & 299 & 32956953120 & 366 & 100522566144 & 444 \\
\hline 1509765120 & 45 & *10200236032 & 96 & 33040072800 & 371 & 103262796000 & 474 \\
\hline 1558745370 & 159 & 10575819520 & 184 & 34174812672 & 239 & 108061356200 & 193 \\
\hline *1630964808 & 99 & 10597041000 & 227 & 34482792960 & 396 & 109111766400 & 474 \\
\hline 1632825792 & 101 & 10597759200 & 357 & *35032757760 & 392 & *109585986048 & 324 \\
\hline 1727271000 & 222 & 10952611488 & 221 & 35793412200 & 371 & ${ }^{*} 110886522600$ & 155 \\
\hline 1862023680 & 158 & 10983408128 & 172 & 37906596000 & 464 & 112202596352 & 176 \\
\hline *1867650048 & 128 & 11076156000 & 322 & 39970476000 & 332 & 115987576320 & 518 \\
\hline 2008725600 & 203 & 11296276992 & 237 & 40053686400 & 464 & *123014892000 & 484 \\
\hline 2140041600 & 188 & 11480905800 & 357 & 40520844000 & 465 & *124406100000 & 375 \\
\hline 2144862720 & 260 & 12941019000 & 229 & 40752391680 & 494 & 126090783000 & 438 \\
\hline 2369162250 & 203 & 13067913600 & 328 & 40805200800 & 369 & 133410461184 & 311 \\
\hline 2481357060 & 201 & 13073550336 & 224 & 42054536160 & 285 & 134369095680 & 89 \\
\hline 2701389600 & 270 & 13398021000 & 328 & 42763096320 & 279 & *137438691328 & 19 \\
\hline 2705020500 & 149 & 13581986600 & 181 & 43783188480 & 87 & 137770869600 & 663 \\
\hline 2716826112 & 228 & 13584130560 & 380 & *43861478400 & 264 & 142275893760 & 398 \\
\hline 2738824704 & 166 & 13660770240 & 169 & 43952044500 & 269 & 142985422944 & 323 \\
\hline 2763489960 & 212 & *14182439040 & 384 & 44184172032 & 309 & 143173648800 & 530 \\
\hline 2777638500 & 255 & 14254365440 & 186 & 45578332800 & 572 & 147112449120 & 367 \\
\hline 2839922400 & 205 & 14378364000 & 440 & 45923623200 & 510 & 150115204512 & 233 \\
\hline *2876211000 & 150 & 14541754500 & 267 & 50497467930 & 303 & 150759100800 & 602 \\
\hline 2945943000 & 218 & 14980291200 & 329 & 51001180160 & 160 & 151955343540 & 373 \\
\hline 3134799360 & 266 & 15174001920 & 264 & 52748186400 & 371 & 153003540480 & 240 \\
\hline 3209343200 & 139 & 15192777600 & 440 & 53227843200 & 334 & 154567413000 & 602 \\
\hline 3221356320 & 195 & 15358707000 & 329 & 53621568000 & 500 & *156473635500 & 390 \\
\hline 3288789504 & 230 & 16003510272 & 53 & 54572427000 & 334 & 156798019840 & 341 \\
\hline 3328809120 & 191 & 16569653760 & 296 & 54648009000 & 285 & 159248314400 & 193 \\
\hline 3349505250 & 205 & 16919229600 & 357 & 56481384960 & 395 & 159381986400 & 531 \\
\hline 3506025600 & 308 & 17624538624 & 253 & *57575890944 & 192 & 164297299320 & 411 \\
\hline 3594591000 & 308 & 18999981000 & 407 & 57629644800 & 384 & 164751121920 & 430 \\
\hline 3702033720 & 213 & ${ }^{*} 19017782784$ & 336 & ${ }^{*} 57648181500$ & 273 & 169696449000 & 295 \\
\hline 3740553180 & 202 & *19209881600 & 256 & 57897151488 & 248 & 169956154368 & 416 \\
\hline 3831421440 & 220 & 19744452000 & 328 & 59388963480 & 402 & *183694492800 & 672 \\
\hline 4143484800 & 312 & 20015559200 & 181 & 61434828000 & 470 & 194743785600 & 611 \\
\hline 4146734592 & 232 & 20387256120 & 391 & 62487000576 & 437 & 201532767744 & 263 \\
\hline 4720896180 & 197 & 21537014400 & 344 & 64834371840 & 282 & *206166804480 & 384 \\
\hline 4738324500 & 261 & 21611457280 & 188 & 64914595200 & 470 & 213815481600 & 405 \\
\hline 5058000640 & 176 & 21943595520 & 392 & ${ }^{*} 66433720320$ & 224 & 217494027520 & 344 \\
\hline 5133201408 & 51 & 22633884000 & 329 & 67622100480 & 302 & 220524885504 & 326 \\
\hline 5275179000 & 226 & 22933532160 & 278 & *71271827200 & 270 & 220920860160 & 515 \\
\hline 5297292000 & 308 & 23450730240 & 272 & *73924348400 & 125 & *221908282624 & 171 \\
\hline 5510647296 & 167 & 23855232960 & 173 & 77120316000 & 472 & 227783556000 & 602 \\
\hline 5579121240 & 214 & 24362612820 & 211 & *77924700000 & 375 & 234605428736 & 184 \\
\hline 5943057120 & 341 & 25559301600 & 369 & 78340298400 & 522 & 236489897160 & 319 \\
\hline 6720569856 & 235 & 25666007040 & 85 & 80422524000 & 334 & 237191556096 & 254 \\
\hline 7279591410 & 163 & 26113432800 & 377 & 80533908000 & 375 & 240423674400 & 534 \\
\hline 7330780800 & 322 & 26242070400 & 456 & 80551516500 & 493 & 250230357000 & 377 \\
\hline 7515963000 & 322 & 26454556800 & 332 & *81417705600 & 484 & *271309925250 & 405 \\
\hline 8104168800 & 351 & 27122823000 & 332 & 81488534400 & 472 & 280541488500 & 505 \\
\hline 8154824040 & 165 & 27689243400 & 369 & 83410119000 & 290 & 285266741760 & 728 \\
\hline 8243595360 & 344 & 27726401736 & 187 & ${ }^{*} 84418425000$ & 375 & 287879454720 & 320 \\
\hline *8410907232 & 171 & 29715285600 & 495 & 87825283840 & 191 & 288662774400 & 836 \\
\hline
\end{tabular}




\begin{tabular}{|c|c|c|c|c|c|c|c|}
\hline$n$ & $H$ & $n$ & $H$ & $n$ & $H$ & $n$ & $H$ \\
\hline 289048687200 & 535 & 753132796416 & 458 & 1578475971072 & 664 & 2915401724928 & 446 \\
\hline 292337717760 & 314 & 765181053000 & 443 & 1584792261000 & 551 & 2965353955200 & 904 \\
\hline 307001350656 & 452 & 779729094144 & 656 & *1599300612000 & 648 & 3076882754400 & 1005 \\
\hline 307030348800 & 462 & 783990099200 & 495 & 1626268644000 & 614 & 3105356994432 & 616 \\
\hline 311203567584 & 333 & 793104238080 & 759 & 1656012758400 & 872 & 3175969724928 & 668 \\
\hline 312402636000 & 478 & 819730138500 & 519 & 1681994012160 & 439 & 3218345676000 & 652 \\
\hline 321300067176 & 197 & *830350521000 & 756 & 1683038945280 & 440 & 3238966130400 & 981 \\
\hline 326196097920 & 736 & 861743282400 & 957 & 1708842189600 & 707 & *3321402084000 & 1080 \\
\hline 330097622400 & 478 & 863638364160 & 416 & 1721209990500 & 715 & 3356538237000 & 389 \\
\hline 336607789056 & 264 & 869516291840 & 366 & 1773515487744 & 471 & 3377333836800 & 847 \\
\hline 341519256000 & 325 & 888875820360 & 327 & 1784852619264 & 372 & 3398177502720 & 776 \\
\hline 349002044160 & 506 & 888988066400 & 277 & 1801169758080 & 762 & 3448576989000 & 545 \\
\hline 350280184800 & 389 & 893835790848 & 658 & 1862961762816 & 612 & 3500961340800 & 946 \\
\hline 362526484320 & 671 & 906550977024 & 331 & 1886043571200 & 516 & 3519081431040 & 460 \\
\hline 384342364800 & 367 & *945884459520 & 756 & 1888271330400 & 699 & 3522876144480 & 675 \\
\hline 403031236608 & 336 & 950432517216 & 339 & 1919938116096 & 463 & 3531726240768 & 488 \\
\hline 405280060416 & 434 & 970956604800 & 888 & 1924339334400 & 729 & 3607776900000 & 725 \\
\hline 410240742912 & 453 & 995024181060 & 401 & 1948245082112 & 191 & *3622293071600 & 245 \\
\hline 417624936960 & 436 & *997978703400 & 279 & 1959868310400 & 1118 & 3634863187200 & 765 \\
\hline 426778934400 & 618 & 1018809792000 & 950 & 1987794251520 & 524 & 3772440804608 & 323 \\
\hline *428440390560 & 546 & *1058501001600 & 648 & 2015156183040 & 560 & 3777406841600 & 530 \\
\hline 428555439000 & 298 & 1070373679200 & 707 & 2020639420800 & 1232 & 3881325763840 & 367 \\
\hline 429520946400 & 689 & 1076349859200 & 614 & 2021976333000 & 555 & *3946161492000 & 735 \\
\hline 434508127200 & 697 & *1085239701000 & 648 & 2033105289600 & 510 & 3962552630400 & 906 \\
\hline 437409004032 & 644 & 1103539437000 & 614 & 2051203714560 & 755 & 3990762504960 & 526 \\
\hline 439655610240 & 744 & 1109541413120 & 285 & 2059445329920 & 434 & 3991394534400 & 858 \\
\hline *443622427776 & 352 & 1135890756000 & 869 & 2061489484800 & 517 & 4029093232640 & 316 \\
\hline 465036042240 & 392 & *1144136294400 & 350 & 2066882988800 & 522 & 4205037804800 & 531 \\
\hline *469420906500 & 507 & 1159571485800 & 707 & 2070303429600 & 729 & 4224973334400 & 1288 \\
\hline 470717137800 & 697 & 1161528261600 & 409 & 2096328767456 & 241 & 4240965560832 & 669 \\
\hline 479411093504 & 188 & 1175104476000 & 899 & *2112394079250 & 585 & *4314435969536 & 385 \\
\hline 482476262400 & 484 & *1179832600464 & 217 & 2128528765440 & 776 & 4346661822720 & 548 \\
\hline 483548738400 & 537 & 1200229430400 & 869 & 2130069916800 & 652 & *4409499089268 & 147 \\
\hline 494122282290 & 317 & 1209584724480 & 584 & 2172650274816 & 284 & 4437102673920 & 464 \\
\hline 502612830720 & 740 & 1211621062400 & 510 & 2183877423000 & 652 & 4517245877760 & 786 \\
\hline 505159855200 & 935 & 1219581548640 & 551 & *2198278051200 & 1080 & 4537735429500 & 754 \\
\hline *513480135168 & 648 & 1233377308800 & 893 & ${ }^{*} 2236152828000$ & 529 & 4603679570880 & 337 \\
\hline 518453342208 & 101 & 1253107608480 & 389 & 2259816300000 & 725 & 4612268729250 & 765 \\
\hline 520212037632 & 272 & 1288623772800 & 622 & 2267834849280 & 704 & 4638285943200 & 1010 \\
\hline 547929930240 & 540 & 1324245491712 & 368 & 2312019021312 & 851 & 4660073935104 & 378 \\
\hline 583096381560 & 422 & 1325481830400 & 736 & 2335483332000 & 725 & 4694568278400 & 1133 \\
\hline 586207480320 & 748 & ${ }^{*} 1330464844800$ & 660 & 2363575441500 & 533 & 4712844296160 & 1001 \\
\hline 603567619200 & 874 & 1331785072800 & 986 & 2439654963200 & 508 & 4713692054400 & 643 \\
\hline 616719527424 & 454 & 1369947647250 & 409 & 2448134325000 & 725 & 4741836503040 & 736 \\
\hline 618269652000 & 860 & 1377031864320 & 432 & 2448278300160 & 781 & 4752162586080 & 565 \\
\hline 626112396000 & 479 & 1386998613000 & 803 & 2468667064500 & 521 & 4824711643136 & 344 \\
\hline 633926092800 & 704 & 1413817996500 & 509 & 2471771484000 & 915 & 4832764209000 & 643 \\
\hline 652482082560 & 516 & 1438233280512 & 282 & 2520477679104 & 621 & 4903097162600 & 361 \\
\hline 653289436800 & 860 & 1447428787200 & 600 & 2567400675840 & 1080 & 4959751305600 & 1296 \\
\hline 661576406400 & 479 & *1480003190400 & 529 & 2608548875520 & 549 & 5085231579136 & 37 \\
\hline 666574634880 & 752 & 1482760097280 & 774 & 2627456832000 & 980 & *5111051997870 & 366 \\
\hline *677701763200 & 340 & 1507838492160 & 962 & 2644660418400 & 979 & 5148385482240 & 758 \\
\hline 693688413600 & 697 & *1517389419000 & 529 & 2677752441000 & 735 & 5268640785408 & 806 \\
\hline 703816286208 & 276 & ${ }^{*} 1542738616320$ & 352 & 2706066874368 & 376 & 5289640356000 & 946 \\
\hline 704575228896 & 405 & *1553357978368 & 252 & 2708593305600 & 752 & 5290460648928 & 629 \\
\hline 713178090240 & 517 & 1556017837920 & 555 & 2708845856640 & 764 & 5431874152320 & 766 \\
\hline 726673802400 & 538 & 1567241676000 & 872 & 2709493768800 & 1003 & 5469709639680 & 608 \\
\hline 726972637440 & 527 & 1571198926080 & 536 & ${ }^{*} 2827553208480$ & 686 & 5681022328800 & 701 \\
\hline
\end{tabular}




\begin{tabular}{|c|c|c|c|c|c|c|c|}
\hline$n$ & $H$ & $n$ & $H$ & $n$ & $H$ & $n$ & $H$ \\
\hline 5745853670400 & 524 & 10410668674560 & 1026 & 17505483899904 & 824 & 27184083544800 & 1041 \\
\hline 5808057260544 & 636 & 10434320851500 & 543 & 17550753948000 & 926 & 27188110404000 & 1224 \\
\hline 5853911263200 & 985 & ${ }^{*} 10461217539500$ & 305 & 17566056012960 & 1066 & 27214447163904 & 1272 \\
\hline 5914045683000 & 457 & 10670692032000 & 995 & 17592306732000 & 1188 & 27258821990400 & 1376 \\
\hline *5914410203520 & 936 & 10680522652800 & 1628 & 18218458487040 & 562 & 27261634143744 & 617 \\
\hline 5956949980800 & 908 & *10711009764000 & 1050 & 18297947606400 & 918 & 27501146956800 & 1140 \\
\hline 6045468549120 & 728 & 10799170314240 & 616 & 18449074917000 & 1224 & 27628679988000 & 919 \\
\hline *6073712944992 & 693 & *10881843388416 & 648 & 18536508900000 & 745 & 27717383688960 & 566 \\
\hline 6175225017000 & 565 & 10996995170304 & 382 & 18544856803200 & 926 & ${ }^{*} 28103080287744$ & 496 \\
\hline 6200648966400 & 783 & 11007262156800 & 764 & 18942468120576 & 658 & 29040286302720 & 1060 \\
\hline 6312101796000 & 878 & 11332220524800 & 795 & 19029577862400 & 801 & 29193612739200 & 919 \\
\hline 6343192620800 & 534 & *11484718245000 & 1125 & 19075764394368 & 688 & 29382474401280 & 1442 \\
\hline 6352588408320 & 554 & 11535568819200 & 526 & 19098061983000 & 1449 & 29495815011600 & 525 \\
\hline 6355147895040 & 542 & *11567890545120 & 1053 & 19172121516800 & 538 & 29646588972000 & 964 \\
\hline 6669629366400 & 878 & 11610780300000 & 745 & 19621667049600 & 964 & 30209639896800 & 1055 \\
\hline 6734495875072 & 49 & 11643511017600 & 1188 & 20193653718784 & 468 & *30233275380000 & 1155 \\
\hline 6764077878600 & 305 & 11725700507136 & 642 & 20432681637984 & 783 & 30368564724960 & 1155 \\
\hline 6793110213120 & 788 & 11810043108864 & 1242 & *20662005324800 & 506 & 30676980297600 & 1336 \\
\hline *68444450880704 & 684 & 11937636711000 & 1188 & 20663813681280 & 1457 & 31094717121000 & 569 \\
\hline 6884622108000 & 916 & 11977778891232 & 765 & 20746479283200 & 946 & 31671732879360 & 828 \\
\hline 7121968308000 & 643 & 11999552292000 & 745 & 21204827804160 & 1115 & 32133029292000 & 1365 \\
\hline 7131668544000 & 1400 & 12087279697920 & 474 & *21590959104000 & 800 & 32176700980480 & 551 \\
\hline 7191166402560 & 470 & 12412499299200 & 1634 & *21733758429600 & 434 & 32327865884160 & 1062 \\
\hline 7274578147200 & 916 & *12452007204000 & 936 & 21738589593600 & 665 & 32713768684800 & 1377 \\
\hline 7318964889600 & 762 & 12493968334848 & 651 & 21755342568960 & 1449 & ${ }^{*} 32752714995000$ & 1155 \\
\hline *7322605472000 & 672 & 12578345325000 & 745 & 21967816416000 & 1008 & 33451592638464 & 664 \\
\hline 7338147328512 & 876 & 12588244300800 & 902 & 22047495446340 & 245 & 34044371361000 & 1476 \\
\hline 7512024199680 & 1106 & 12602388395520 & 1035 & 22051566231552 & 383 & 34222225403520 & 1140 \\
\hline 7531474204800 & 1312 & 12757657068800 & 537 & 22072153958400 & 766 & 34854206521344 & 536 \\
\hline 7574491607040 & 173 & 12876333500800 & 646 & 22332001910400 & 1702 & 35085648124800 & 1337 \\
\hline 7626085510400 & 535 & 13202304998400 & 903 & *22385029489560 & 198 & 35137010809600 & 986 \\
\hline 7741979148288 & 506 & 13217359034880 & 1112 & 22717860433632 & 657 & 35195158303200 & 1377 \\
\hline 7761092320800 & 1014 & 13230227556000 & 662 & 22735712876800 & 957 & 35560552416480 & 1079 \\
\hline 7766789891840 & 420 & 13327831686400 & 935 & 22742476922880 & 632 & 35727233502464 & 483 \\
\hline 7780605009408 & 639 & 13552871623200 & 1038 & 23300369675520 & 630 & 36457089596928 & 1278 \\
\hline 7867987832250 & 783 & ${ }^{*} 13661860101120$ & 1056 & 23375124208800 & 1018 & 36501751345920 & 563 \\
\hline *8449576317000 & 936 & 13914857829600 & 1313 & 23409541693440 & 816 & 36567846174720 & 478 \\
\hline 8467093071360 & 1022 & 14115958857000 & 1428 & 23814974355480 & 802 & 36690736642560 & 1460 \\
\hline 8468207666688 & 514 & 14379426038250 & 795 & 23819044650240 & 557 & 36783914076000 & 1242 \\
\hline 8633641161600 & 1316 & 14474134929408 & 516 & *23885971200000 & 960 & 37342487131488 & 795 \\
\hline 8729162297856 & 1224 & 14635113292800 & 1210 & 23906526134400 & 911 & 37643864076000 & 929 \\
\hline 8756458300800 & 662 & *14747907505800 & 434 & 23929031075040 & 569 & 37695962304000 & 1850 \\
\hline 8867577438720 & 785 & 14814719631360 & 446 & 24133566352896 & 1269 & 38287967477760 & 1064 \\
\hline 8924263096320 & 620 & 14873771827200 & 650 & 24146583347250 & 801 & 38583480499200 & 1276 \\
\hline 8977654413000 & 662 & 15007087898880 & 989 & 24345523036800 & 1242 & 38629000502400 & 969 \\
\hline 9027208888320 & 472 & 15147350507520 & 792 & *24613169545216 & 285 & 38781262840320 & 1476 \\
\hline 9068974548480 & 789 & 15246642902400 & 1328 & 24722083685376 & 854 & 38903025047040 & 1065 \\
\hline 9231944494500 & 767 & 15337823806032 & 403 & 24960513123000 & 1242 & 39275901181440 & 1474 \\
\hline 9269718441984 & 644 & *15462510336000 & 960 & 25075635936512 & 339 & 39377859655680 & 764 \\
\hline 9314808814080 & 1020 & 15820566085632 & 517 & 25206921653760 & 1462 & 40220975692800 & 917 \\
\hline 9564679210240 & 671 & 15889967976960 & 1044 & 25278832051200 & 1254 & 40369640927616 & 1144 \\
\hline 9689839810560 & 752 & 16080035811840 & 1428 & 25483518950400 & 913 & 40815295466400 & 1042 \\
\hline *9831938337200 & 350 & 16212258972000 & 942 & 26025228028800 & 1142 & 40963871894400 & 1338 \\
\hline 10112079035520 & 1426 & 16524280700928 & 656 & 26183184168960 & 762 & 41051610243072 & 713 \\
\hline 10132001510400 & 1050 & *16924847940000 & 1125 & 26407085632256 & 476 & 41975434828800 & 1160 \\
\hline 10256659997220 & 421 & 16965637957800 & 527 & 26757162432000 & 998 & 42848120544768 & 1173 \\
\hline *10297226649600 & 630 & 17086937762048 & 462 & 26772789288960 & 806 & *43180427911400 & 403 \\
\hline 10341947847528 & 373 & 17130547324800 & 942 & 26818992224640 & 1464 & 43588078934400 & 1661 \\
\hline
\end{tabular}




\begin{tabular}{|c|c|c|c|c|c|c|c|}
\hline$n$ & $H$ & $n$ & $H$ & $n$ & $H$ & $n$ & $H$ \\
\hline 43645811489280 & 2040 & 54942374462976 & 1284 & 68029152998400 & 1175 & 81178611180800 & 1005 \\
\hline 43865704602720 & 1331 & 55062424216800 & 1853 & 68919093243000 & 1494 & *84761657875440 & 651 \\
\hline 43905339878400 & 1573 & 56087667603968 & 715 & 69775118828800 & 979 & 84762932436000 & 1272 \\
\hline *43947421401888 & 216 & 56100553084800 & 1272 & 70879832150400 & 1752 & 85372015528800 & 1859 \\
\hline 44008577613000 & 1484 & 56221571976570 & 671 & 70902132973056 & 647 & 85398298444800 & 1180 \\
\hline 44095620366336 & 524 & 56261841199200 & 1059 & 71485642681600 & 1003 & 85434688810240 & 770 \\
\hline 44345330883840 & 1037 & 56463835428000 & 2040 & 71681373036000 & 1769 & 85454920812800 & 981 \\
\hline 44531496801792 & 886 & 56483529822720 & 794 & 71969788628304 & 427 & 85626151750656 & 722 \\
\hline 44621315481600 & 900 & 57209988326400 & 1290 & 72370674647040 & 860 & 86100192346112 & 367 \\
\hline 45108496097280 & 776 & 57516364550400 & 807 & 72874680721920 & 1512 & 86887495094400 & 2408 \\
\hline 45124517299680 & 1073 & 57517704153000 & 1272 & 729823698 & 807 & 87548446375936 & 91 \\
\hline 454060181 & 670 & 5862850 & 1070 & 7298268 & 848 & 885278 & 526 \\
\hline 45634960425600 & 1739 & 586373136 & 1073 & 732 & 479 & 8884750 & 1498 \\
\hline *46013471418096 & 558 & 58954886991000 & 1491 & 74454619599360 & 2088 & 88954751508480 & 824 \\
\hline *46353444300800 & 760 & 5896005339 & 986 & 7515357 & 1278 & 89163516169728 & 887 \\
\hline 46796172040800 & 1019 & *58991630023200 & 620 & 75167128 & 1269 & 89229599 & 1943 \\
\hline 47089809930800 & 455 & 59050215544320 & 2070 & 76343936628960 & 1323 & 89875965763584 & 669 \\
\hline 47824897105920 & 682 & 59888894456160 & 1275 & 76392247932000 & 2070 & *90134334505600 & 952 \\
\hline *47911115564928 & 1008 & 60580961156352 & 702 & 77052018771000 & 1278 & 90606219580800 & 1202 \\
\hline 48765763791360 & 1068 & 60868244209500 & 778 & 77212128389760 & 1368 & 91501705658880 & 626 \\
\hline 49749547075200 & 1269 & 60876700907400 & 549 & 77547710691360 & 1086 & 92895015213000 & 1202 \\
\hline 49921679808000 & 1862 & 61015386432000 & 1540 & 77654820789000 & 1421 & 92945339487000 & 1716 \\
\hline 50131876354560 & 1484 & 61259428298250 & 1131 & 78429196876800 & 1331 & 94258317081600 & 921 \\
\hline 50560395177600 & 2070 & 61986015974400 & 759 & 78497425843200 & 1298 & 96320660436000 & 2088 \\
\hline 51006265947000 & 1269 & 62469841674240 & 1085 & 78508410140160 & 1494 & 96422831210496 & 548 \\
\hline 51260813286144 & 693 & 62707195371744 & 801 & 78730921315200 & 967 & 97516898519040 & 1376 \\
\hline 51633280258560 & 1448 & 63687677113088 & 492 & 78958268284896 & 1287 & 97769262366720 & 852 \\
\hline 52500435423744 & 523 & 63750063484800 & 2088 & 78961886115840 & 766 & 97789867812000 & 947 \\
\hline *53092467020880 & 651 & 64720497623040 & 846 & 79221256896768 & 714 & 98079457512960 & 1074 \\
\hline 54409216942080 & 1080 & 65058512238720 & 1716 & *79708161843200 & 660 & & \\
\hline 54557264361600 & 2376 & 67157796625920 & 1491 & 80508613785600 & 1397 & & \\
\hline *54934276752360 & 252 & 67306216513536 & 668 & 80548660192000 & 1232 & & \\
\hline
\end{tabular}

\section{References}

[ 1 ] J. Brillhart, D.H. Lehmer, J.L. Selfridge, B. Tuckerman and S.S. Wagstaff Jr., Factorizations of $b^{n} \pm 1, b=2,3,5,6,7,10,11,12$ up to high powers, 3rd edition. Contemp. Math., 22, American Mathematical Society, Providence, RI, 2002, 1-13.

[ 2 ] G.L. Cohen, Numbers whose positive divisors have small integral harmonic mean. Math. Comp., 66 (1997), 883-891.

[ 3 ] G.L. Cohen and R.M. Sorli, Harmonic seeds. Fibonacci Quart., 36 (1998), 386-390; Errata. Fibonacci Quart., 39 (2001), 4.

[4] M. Garcia, On numbers with integral harmonic mean. Amer. Math. Monthly, 61 (1954), 89-96.

[ 5 ] T. Goto and S. Shibata, All numbers whose positive divisors have integral harmonic mean up to 300. Math. Comp., 73 (2004), 475-491.

[6 ] R.K. Guy, Unsolved Problems in Number Theory, 3rd edition. Springer-Verlag, New York, 2004.

[7] O. Ore, On the averages of the divisors of a number. Amer. Math. Monthly, 55 (1948), 615-619.

[ 8 ] R.M. Sorli, Algorithms in the Study of Multiperfect and Odd Perfect Numbers. Ph.D. thesis, University of Technology, Sydney, 2003. 